\title{
Magnitude and Associated Factors of Liver Fibrosis among Mono- Infected HIV Patient Using Noninvasive Marker Aspartate Transaminase to Platelet Ratio Index
}

\author{
Yemanebrhane $\mathrm{N}^{1 *}$, Melese $\mathrm{D}^{1}$, Liknew $\mathrm{W}^{1}$, Andargachew $\mathrm{G}^{1}$, \\ Desale $\mathrm{A}^{1}$ and Sisay $\mathrm{A}^{2}$ \\ 1Ethiopian Public Health Institute, Ethiopia \\ ${ }^{2}$ Addis Ababa University, School medical laboratory, Ethiopia
}

Research Article

Volume 2 Issue 2

Received Date: June 05, 2018

Published Date: June 13, 2018

*Corresponding author: Nebiyou Yemanebrhane, Ethiopian Public Health Institute, Ethiopia, Tel: +251 910089081 ;

Email: nebilyma@gmail.com

\section{Abstract}

Background: Liver disease is one of the primary causes of morbidity and mortality among human immunodeficiency virus (HIV) infected patients. These HIV patients are at risk of liver fibrosis. Diagnose of liver fibrosis using noninvasive markers among HIV infected people in sub-Saharan Africa including Ethiopia are not widely used in order to perceive patient outcome.

Method: A hospital based cross sectional study was conducted at Zewditu Memorial Hospital. Participants' sociodemographic and clinical information were collected from the Anti-Retroviral Therapy (ART) database. Blood samples were collected from 195 volunteer, patients taking HAART for at least 1 year. The APRI score were used to determine the presence of significant fibrosis. Data was entered and analyzed by SPSS version 20 and $p$ value less than 0.05 was taken as statistically significant.

Result: Of the 195 participants, majority of study participants were in age group 30-39 (45.12\%), female (57.43\%) and they were married (49.22\%).Overall burden of liver fibrosis by APRI index was $4.09 \%$. On the multivariate analysis after adjusting for age, CD4, WHO staging, BMI, being male (adjusted odds ratio $=0.122 ; 95 \%$ CI: $0.017-0.883$ ) were significantly associated factors of liver fibrosis as measured by APRI.

Conclusion: Significant fibrosis by APRI score was found in 4.09\% among of HIV mono-infected patients. Male sex was associated with significant fibrosis. Further studies shall be conducted to consolidate these study findings.

Keywords: APRI; CD4; HIV; Liver Fibrosis

Magnitude and Associated Factors of Liver Fibrosis among Mono-Infected HIV Patient Using 


\section{Journal of Infectious Diseases \& Travel Medicine}

\section{Introduction}

Liver disease is one of the primary causes of morbidity and mortality among human immunodeficiency virus (HIV) patients. These HIV patients are at risk of liver fibrosis. To diagnose liver fibrosis Liver biopsy is gold standard method, however these techniques have its own drawback: may cause complications, invasiveness, is costly, and is limited by sampling errors. Hence, noninvasive markers have been proposed and from other noninvasive markers of liver fibrosis, Aspartate to platelet ratio index (APRI) has been shown as an easy and inexpensive marker of liver fibrosis. APRI has also recently been used to determine advanced fibrosis in HIVmonoinfected patients with high value $[1,2]$.

Highly active anti-retroviral therapy (HAART) has transformed HIV from a uniformly fatal illness into a manageable chronic infection and has been shown to be able to restore CD4+ T cells in HIV infected patients, however these therapy cause liver fibrosis in most of HAART taking patient after long term treatment [3].

To diagnose liver fibrosis among HIV infected people living in sub-Saharan Africa noninvasive markers are not widely used in order to perceive patient outcome. Moreover, data on usage and practice of non invasive liver fibrosis markers are limited in sub-Saharan Africa. These study investigated burden of hepatic fibrosis using APRI score which is one of noninvasive marker with good specificity and sensitivity among mono-infected HIV patients and its association with socio demographic data.

\section{Material and Methods}

A cross sectional study was conducted in Zewditu memorial hospital (ZMH) ART clinic. According to the annual report of the Addis Ababa City Administration Health Bureau for July 2015, there were a total of 125,994 HIV positive clients (94.4\% adults) who ever enrolled to ART services, among which 74,986 (94.8\% adults) had ever started ART and there were 53,677 (95.5\% adults) currently on ART. The city in 2015, 11 public hospitals, 77 Government health centres, 14 private hospitals, and 9 Non Government clinics provided ART services in Addis Ababa. So the present study was conducted at Zewditu Memorial hospital, which is one of referral public health hospital in Addis Ababa, with strong HIV /AIDS care and treatment centre.

The Hospital is one of PEPFAR funded in Ethiopia, largest and most comprehensive HIV care and treatment site. It was selected as the first pilot location for HIV programs and grew into a model program. In July 2003, Ethiopia's first ART program started at Zewditu with support of CDC-Ethiopia, and served HIV positive patients from around the country for a nominal fee. Through PEPFAR's support, the hospital began a free ART program in March 2005. Since the Hospital, one of the referral hospitals for the surrounding sub city with wide catchment area, most patients have follow up in this hospital. There are a total of 10,672 patients enrolled to HIV

Socio-demographic information and other important possible risk factors of the study participants was routinely collected from the ART database and from their medical card using structured data collection sheet. Eight $\mathrm{ml}$ of venous blood samples were aseptically collected using plain and EDTA vacutainer tubes ( $4 \mathrm{ml}$ in each tube) for the determination of CD4, PLT and AST levels from each study participants. The blood specimen in the plain tube was centrifuged at 3000 RPM for 5 minutes to separate the serum and used for determination of AST within one hour of separation. The second tube that contains whole blood was used for the CD4 and PLT levels determination. TheCD4 count was determined by BD FACS calibur flowcytometry (Beckton Dickinson, San Jose, California). AST were analyzed using Humastar 200 (Human GmbH, Weisbaden, Germany).

The APRI score is defined as: (AST [/ ULN]*100/platelet count [10\%/L]) [4]. An APRI score $>1.5$ has been shown to be predictive of significant liver fibrosis (defined as an Ishak fibrosis score $>3$ ) with an area under the receiver operating characteristic curve of 0.76-0.85 [4-6]. Thus, we used the established cut off of 1.5 to determine the presence of significant fibrosis.

\section{Quality Assurance}

To ensure the quality of data, all the laboratory investigations were performed following standard operating procedures. The CD4 count, AST and PLT tests were carried out in nationally and ISO 15189 internationally accredited laboratories.

External Quality Assurance feedbacks of the laboratories were acceptable in addition to this internal quality controls were run before running study samples. There was a regular supervision to data collectors by the principal investigators to maintain the data quality. Completeness of the data was declared through periodic data collection. Finally data was analyzed employing appropriate statistical treatment following proper entry and cleaning. 


\section{Journal of Infectious Diseases \& Travel Medicine}

\section{Data Processing and Analysis}

All data were analyzed using SPSS version 20 software for windows. Differences between patients with and without significant fibrosis by APRI score were assessed using Chi-square and Multivariable logistic regression was used to determine risk factors for significant fibrosis by APRI score.

\section{Result}

Majority of study participants were in age group 30-39 $(45.12 \%)$, female $(57.43 \%)$ and they were married (49.22\%).In line with these Table 1 result shows that age category and HAART combination of mono-infected HIV patient and APRI are significantly related $(p<0.05)$. Moreover obese category of body mass index was at higher number of liver fibrosis relative to other groups of body mass index even if it was not statically significant.

\begin{tabular}{|c|c|c|c|c|}
\hline \multicolumn{2}{|c|}{ Variable } & APRI $\leq 1.5$ & APRI $\geq 1.5$ & $P$-value \\
\hline \multirow{4}{*}{ Age } & $18-29$ & $\frac{\mathbf{N}(\%)}{14(7.17)}$ & $\frac{\mathbf{N}(\mathbf{\%})}{4(2.05)}$ & \multirow{4}{*}{$0.001^{*}$} \\
\hline & $30-39$ & $86(44.1)$ & $2(1.02)$ & \\
\hline & $40-49$ & $51(26.15)$ & $2(1.02)$ & \\
\hline & $>50$ & $36(18.46)$ & $0(0)$ & \\
\hline \multirow{2}{*}{ Sex } & M & $77(39.48)$ & $6(3.07)$ & \multirow{2}{*}{0.06} \\
\hline & $\mathrm{F}$ & $110(56.41)$ & $2(1.02)$ & \\
\hline \multirow{5}{*}{ Marital status } & Single & $40(20.51)$ & $3(1.53)$ & \multirow{5}{*}{0.3} \\
\hline & Married & $93(47.69)$ & $3(1.53)$ & \\
\hline & Divorced & $24(12.3)$ & $2(1.02)$ & \\
\hline & Widowed & $30(15.38)$ & $2(1.02)$ & \\
\hline & Under weight & $27(13.84)$ & $1(0.51)$ & \\
\hline \multirow{3}{*}{ BMI } & Normal & $98(50.25)$ & $2(1.02)$ & \multirow{3}{*}{0.19} \\
\hline & Obese & $49(25.12)$ & $3(1.53)$ & \\
\hline & Overweight & $13(6.67)$ & $2(1.02)$ & \\
\hline \multirow{2}{*}{$\mathrm{CD} 4$} & $<200 \mathrm{cell} / \mathrm{cumm}$ & $20(10.25)$ & $2(1.02)$ & \multirow{2}{*}{0.21} \\
\hline & $>200 \mathrm{cell} / \mathrm{cumm}$ & $167(85.64)$ & $6(3.07)$ & \\
\hline \multirow{8}{*}{ HAART } & 1c(AZT-3TC-NVP) & $30(15.380$ & $2(1.02)$ & \\
\hline & 1d( ZT-3TC-EFV) & $55(28.20)$ & $2(1.02)$ & \multirow{7}{*}{$<0.0001^{*}$} \\
\hline & 1e( TDF-3TCEFV) & $59(30.25)$ & $1(0.51)$ & \\
\hline & $1 \mathrm{f}(\mathrm{TDF}+3 \mathrm{TC}+\mathrm{NVP})$ & $39(20)$ & $1(0.51)$ & \\
\hline & $1 \mathrm{~g}(\mathrm{ABC}+3 \mathrm{TC}+\mathrm{EFV})$ & $0(0)$ & $2(1.02)$ & \\
\hline & 2e(AZT-3TC-LPV/r) & $1(0.51)$ & $0(0)$ & \\
\hline & 2h(TDF-3TC ATV/r) & $1(0.51)$ & $0(0)$ & \\
\hline & $2 \mathrm{i}(\mathrm{ABC}+3 \mathrm{TC}+\mathrm{LPV} / \mathrm{r})$ & $2(1.02)$ & $0(0)$ & \\
\hline \multirow{4}{*}{ WHO stages } & 1 & 31 & 0 & \multirow{4}{*}{0.47} \\
\hline & 2 & 43 & 2 & \\
\hline & 3 & 75 & 5 & \\
\hline & 4 & 38 & 1 & \\
\hline
\end{tabular}

Table 1: Baseline subject characteristics, overall and by significant fibrosis as determined by AST-to-platelet ratio index (APRI) score (APRI >1.5). 


\section{Journal of Infectious Diseases \& Travel Medicine}

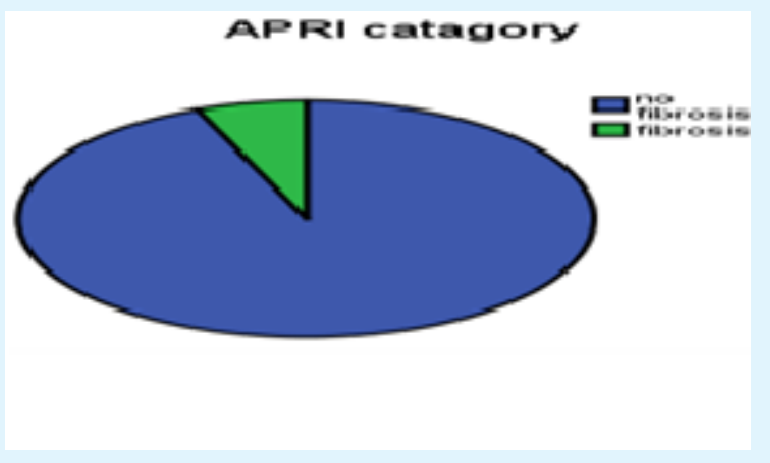

Figure 1: Inference of liver fibrosis using aspartate transaminase to platelet ratio index among HAART taking HIV infected patient, 2017.

On the multivariate analysis after adjusting for age, $\mathrm{CD} 4$, WHO staging, BMI, being male (adjusted odds ratio = 0.122; 95\% CI: 0.017- 0.883) were significantly associated factors of liver fibrosis measured by APRI. As shown in Table 2, female study participant are less likely to have liver fibrosis (Table-2).

\begin{tabular}{|c|c|c|c|c|c|}
\hline \multicolumn{2}{|c|}{ Variables } & Unadjusted odds ratio (CI 95\%) & P value & Adjusted odds ratio (CI $95 \%$ ) & $P$ value \\
\hline \multirow{2}{*}{ Sex } & Female & 1 & & 1 & \\
\hline & Male & $0.233(0.046-1.187)$ & 0.08 & $0.122(0.017-0.883)$ & 0.037 \\
\hline \multirow{4}{*}{ Age Category } & $18-29$ & 1 & & 1 & \\
\hline & $30-39$ & $1.68(0.90-2.24)$ & 0.089 & $1.082(0.49-2.34)$ & 0.841 \\
\hline & $40-49$ & $1.09(0.54-2.16)$ & 0.802 & $0.93(0.40-2.16)$ & 0.876 \\
\hline & $>50$ & $2.29(0.99-5.81)$ & 0.052 & $1.44(0.51-4.00)$ & 0.483 \\
\hline \multirow{4}{*}{ BMI } & Underweight & 1 & & 1 & \\
\hline & Normal & $0.241(0.020-2.904)$ & 0.262 & $0.118(0.005-3.008)$ & 0.196 \\
\hline & Obese & $0.133(0.017-1.024)$ & 0.053 & $0.182(0.016-2.037)$ & 0.167 \\
\hline & Overweight & $0.398(0.060-2.636)$ & 0.34 & $0.639(0.063-6.439)$ & 0.704 \\
\hline \multirow{2}{*}{ CD4 } & $>200 \mathrm{cell} / \mathrm{cumm}$ & 1 & & 1 & \\
\hline & $<200 \mathrm{ell} / \mathrm{cumm}$ & $2.783(0.526-14.729)$ & 0.229 & $1.452(0.125-16.898)$ & 0.766 \\
\hline \multirow{4}{*}{ WHO stage } & 1 & 1 & & 1 & \\
\hline & 2 & $0.489(0.091-8.611)$ & 0.819 & $0.481(0.012-8.61)$ & 0.901 \\
\hline & 3 & $1.767(0.154-20.274)$ & 0.647 & $0.758(0.042-13.622)$ & 0.851 \\
\hline & 4 & $2.533(0.286-22.460)$ & 0.404 & $1.905(0.168-21.62)$ & 0.603 \\
\hline
\end{tabular}

Table 2: Multivariate analysis of Factors associated with liver fibrosis at Zewditu Memorial Hospital, ART clinic, 2017.

\section{Discussion}

To best of our knowledge, systemic assessment of liver fibrosis using non-invasive marker among HIV infected persons has not been reported from Ethiopia. Aspartate transaminase to platelet ratio index (APRI) is an easy way determines liver fibrosis. These study resulted in $4.09 \%$ of HIV infected Ethiopian adults had significant hepatic fibrosis or cirrhosis during therapy. These patients were at much higher risk for mortality. This indicate the need for liver fibrosis screening at the time of HAART initiation and throughout therapy time in line with CD4 and other basic organ function tests.

The prevalence of significant liver fibrosis among HIV mono-infected patient is lower than study by Sulkowski, et al., Dallapiaza, et al., and Gitau, et al., which was 7\% ,8.3\% and7.1\% respectively [7-9]. However, the present study prevalence of significant liver fibrosis is higher than study by Price et al (2012) which was 3.5\% [2]. This 


\section{Journal of Infectious Diseases \& Travel Medicine}

variability for prevalence among studies could be due to usage of different upper limit value for AST since we use APRI for determination of liver fibrosis. In addition to these study participant ethnicity difference and other socio demographic variation could also be the possible reason for prevalence difference.

Our study showed that HIV patients who are on HAART have $<200$ cell /cumm were not significantly exposed to liver fibrosis as measured by APRI. These study findings supported by study in Philadelphia by Dallapiazza, et al., (2010) [8]. However, it was opposed by Price, et al., (2012) which showed that being $C D 4<200$ were independently associated with higher APRI value [2].

The present study reveals that male sex was more likely exposed to liver fibrosis than female based on APRI score. This study result supported by studies in Uganda (2011) which showed that male gender[10].whereas study by Dallpiaze, et al., in 2012 were showed that being male was not risk factor for significant liver fibrosis [8]. The possible reason for these controversial outcomes between different studies could be due to the fact that using of variable sample size, different ethnicity and location of study, and different drugs other than HAART.

\section{Conclusion}

Significant fibrosis by APRI score was found in $4.09 \%$ among of HIV mono-infected patients. Male sex was associated with significant fibrosis. Further studies shall be conducted to consolidate these study findings.

\section{Declarations}

\section{Ethics Approval and Consent to Participant}

Ethical Approval: The study was conducted after being ethically cleared by Department of Medical Laboratory Sciences of Addis Ababa University and Addis Ababa Regional Health Bureau. Informed consent was obtained from the study participants after explaining the aim of the study including their right to withdraw from the study without compromising their care in the hospital. Confidentiality of data was maintained throughout the study.

All results and information obtained from the study was kept confidential at all times in the lockable cabinet.

\section{Authors' Contributions}

Nebiyou Yemanebrhane conceived and designed the study and collected data, performed analysis, interpretation of data and also critically reviewed of this manuscript.

\section{Acknowledgment}

First of all we would like to thank to our almighty GOD who giving us good health throughout our life. We also thankful ZMH, Addis Ababa Health Bureau and Respective Hospital Laboratories for giving the ethical approval visited by this study. Our last but not least gratitude goes to our study participants who gave their time and knowledge to provide all the necessary information, friends and families for all the support during this study.

\section{References}

1. Moodie EE, Pant Pai N, Klein MB (2009) Is antiretroviral therapy causing long term liver damage? A comparative analysis of HIV-monoinfected and HIV/hepatitis C co-infected cohorts. PLoS One 4(2): e4517.

2. Price JC, Seaberg EC, Badri S, Witt MD, D'Acunto K, et al. (2012) HIV Monoinfection Is AssociatedWith Increased Aspartate Aminotransferase-to Platelet Ratio Index, a Surrogate Marker for Hepatic Fibrosis. J Infect Dis 205(6): 1005-1013.

3. Abera B, Zenebe Y, Mulu W, Kibret M, Kahsu G (2014) Seroprevalence of hepatitis B and $C$ viruses and risk factors in HIV infected children at the Felgehiwot Referral Hospital, Ethiopia. BMC Res Notes 7: 838.

4. Wai CT, Greenson JK, Fontana RJ, Kalbfleisch JD, Marrero JA, et al. (2003) A simple noninvasive index can predict both significant fibrosis and cirrhosis in patients with chronic hepatitis C. Hepatology 38 (2): 518-526.

5. Kelleher TB, Mehta SH, Bhaskar R, Sulkowski M, Astemborski J, et al. (2005) Prediction of hepatic fibrosis in HIV/HCV co-infected patients using serum fibrosis markers: the SHASTA index. J Hepatol 43(1): 78-84.

6. Nunes D, Fleming C, Offner G, O'Brien M, Tumilty S, et al. (2005) HIV infection does not affect the performance of noninvasive markers of fibrosis for the diagnosis of hepatitis $C$ virus-related liver disease. J Acquir Immune Defic Syndr 40(5): 538-544.

7. Sulkowski MS, Mehta S, Montes de Occa R, Moore RD, Thomas DL, et al. (2006) Estimated prevalence of significant liver disease among 4,052 HIV infected 
adults with and without chronic hepatitis B and C. $13^{\text {th }}$ Conference on Retroviruses and Opportunistic Infections, Denver, CO, USA.

8. Dalla Piazza M, Amorosa VK, Localio R, Kostman JR, Re VL, et al. (2010) Prevalence and risk factors for significant liver fibrosis among HIV-monoinfected patients. BMC infectious diseases 10(1): 116.

9. Gitau SN, Vinayak S, Silaba M, Adam R, Shah R, et al. (2016) High Prevalence of Liver Fibrosis in Patients with Human Immunodeficiency Virus Monoinfection and Human Immunodeficiency Virus Hepatitis-B Coinfection as Assessed by Shear Wave Elastography: Study at a Teaching Hospital in Kenya. J clin imaging sci 6: 22

10. Stabinski L, Reynolds SJ, Ocama P, Laeyendecker O, Boaz I, et al. (2011) High prevalence of liver fibrosis associated with HIV infection: a cross-sectional study in rural Rakai, Uganda. Antiviral therapy. 16(3): 405411. 